\title{
Mathematical Modeling of Workflows in Production Systems
}

\author{
Stroiteleva T. G. ${ }^{1} \&$ Vukovich G. G. ${ }^{2}$ \\ ${ }^{1}$ Doctor of economics, Professor, Dean of the Faculty of Economics ANOO VO, Altai Academy Economics and \\ Law, Russia \\ ${ }^{2}$ Doctor of economics, Professor, Head. Department of Applied Economics and Management staff Ulianov, \\ Kuban State University, Russia \\ Correspondence: Stroiteleva T. G., Doctor of economics, Professor, Dean of the Faculty of Economics ANOO \\ VO "Altai Academy Economics and Law, Russia. E-mail: stroiteleva_tg@mail.ru
}

Received: October 24, 2014

Accepted: December 28, 2014 Online Published: January 11, 2015

doi:10.5539/mas.v9n3p173

URL: http://dx.doi.org/10.5539/mas.v9n3p173

\begin{abstract}
This article proposes a new method of modeling active elements in production systems, including formulation of management tasks in hierarchical active systems in the circumstances of unidentifiability of reactions of the lower levels (workers); offers a game-theoretic model of active systems of corrective type; studies the mathematical models of incentives for the personnel in workflows; carries out parameters identification and validation of the basic and applied models; as well as develops a set of specific mathematical models to stimulate the performers of workflows.
\end{abstract}

Keywords: mathematical modeling, decomposition models, workflows, production systems, basic model, hierarchical systems

\section{Introduction}

The article introduces a theoretical model of personnel management in production systems in the circumstances of unidentifiable reaction of performers, and the research on its basis of a set of problem-oriented decomposition models and methods in single-stage production systems. It develops a game-theoretic model of active corrective systems, studies the mathematical models of incentives for staff in the workflows, identifies the parameters and validates the adequacy of the basic and applied models (Bogoviz \& Stroiteleva, 2012; Dosi \& Teece, 1998).

\section{Methodology}

The main research goal is development, which uses methods of workflows decomposition.

\subsection{Theoretical Problems of Modeling Workflows}

In the development of a game-theoretic model of corrective optimization, the methods of its particularization and practical application are examined, the complex systems are analyzed as objects of personnel management, and the setting of targets of institutional arrangements synthesis is suggested. As a result, we obtain a new basic model of a hierarchical system, which is written on the basis of the corrective optimization task (Oskorbin, 1987; Freeman, 1988). The results presented here are of a methodological nature.

Implementation of this methodological approach is carried out using the mathematical model of management the corrective optimization model. Let us write down this model for one-step hierarchical systems in an environment, where the vector of parameters (disturbances) of the system $\omega$ is known to the performer, but the management center does not know it.

Let $x^{*} \in X(\omega)$ be the optimal solution in a one-step hierarchical system, wherein $\omega \in \Omega ; f(x, \omega)$ is the objective function of solution selection, determining the income from the operation of the production system during the planning period. Below, we will consider the objective function, which characterizes the income flow and determines the growth rate of the controlled system's value within the accuracy to the parameters.

Let us assume that the choice of the solution is carried out in two stages. At the first stage, the management center without knowing the value of the parameter $\omega$ selects the preliminary solution: $x^{0} \in X^{0}$. We will define the set of preliminary selection of the decision $X^{0}$ below.

At the second stage, the performer (the lower level of the system), knowing the preliminary solution $x^{0}$ and the 
values of the parameters vector $\omega$ specifies (corrects) the solution $x^{0}$ to the value $x^{K} \in X(\omega)$. At that, the overall effectiveness of the system operation may rise as well as fall. The task of the synthesis of the personnel management mechanisms is to ensure the maximum possible increase in the efficiency of the production system's (an industrial enterprise's) operation. The complexity of this problem consists in that the management center has limited awareness of the operating conditions of the lower level.

Let us write down the general mathematical form of the problem of choice of solutions in the considered conditions. To select the preliminary solution, one must switch on $x^{0} \in X(\omega)$ for all $\omega \in \Omega$ and provide for high initial efficiency of the system, since some components of the solution cannot be adjusted. These requirements are met by the following task:

$$
\begin{aligned}
& \bar{f}\left(x^{0}\right)=\max _{x \in X^{0}} \bar{f}(x), \text { wherein } x^{0}=\bigcap_{\omega \in \Omega} X(\omega) ; \\
& \bar{f}(x)=\inf _{\omega \in \Omega} f(x, \omega) .
\end{aligned}
$$

The problem (1) of the choice of solutions at the preliminary stage can be difficult, and in some cases impracticable (for example, if $x^{0}$ is an empty set). In such a situation, other formulations of tasks of searching for a preliminary solution in conjunction with the operation of the prior solution designing for the set $X(\omega)$ are used. In addition, to assess accurately the working conditions of performers, evaluation of the preliminary solution's effectiveness is required, which may be obtained at the end of the planning period.

Let us consider the problem of making decisions in the second stage. Let us introduce the index of the maximum possible effect of correction of a preliminary solution $\bar{\Delta}\left(x^{0}, \omega\right)$ for any $\omega \in \Omega$ :

$$
\bar{\Delta}\left(x^{0}, \omega\right)=\max _{x \in X(\omega)} f(x, \omega)-f\left(x^{0}, \omega\right), \quad \bar{\Delta}\left(x^{0}, \omega\right) \geq 0 .
$$

If for some $\omega \in \Omega$ the inequality (2) will be strict, then the performer can increase the optimization result. He will be keen to perform this function, as the incentive will be more than the labor input for correcting the preliminary solution. It should be noted that the maximum possible effect of correcting the solutions corresponds to the optimal solution $x^{*} \in X(\omega)$ in the considered hierarchical system.

Let $\tilde{\Delta}\left(x, x^{0}, \omega\right)=f(x, \omega)-f\left(x^{0}, \omega\right), x \in X(\omega)$ be the value of income in the system per unit of time due to correction of the preliminary solution. Using the index $\sigma \in[0-1]$, let us denote the income distribution factor from correction of the solution between the management center (the interests of the system as a whole) and the performer (the interests of the low-level management, as well as private interests). Then, the problem of the second stage is as follows:

1. The management center's task:

$$
M^{0}(\sigma, x)=(1-\sigma) \tilde{\Delta}\left(x, x^{0}, \omega\right) \rightarrow \max _{\sigma \in[0-1]}
$$

2. The performer's task:

$$
M(\sigma, x)=\sigma \tilde{\Delta}\left(x, x^{0}, \omega\right)-\varphi\left(\tilde{\Delta}\left(x, x^{0}, \omega\right)\right) \rightarrow \max _{x \in X(\omega)},
$$

wherein $\varphi\left(\tilde{\Delta}\left(x, x^{0}, \omega\right)\right)$ is the function of labor input of the performer in the processes of correcting the preliminary solution, which as an assumption depends only on the values of the function $\tilde{\Delta}\left(x, x^{0}, \omega\right)$.

Thus, the hierarchic system of decision-making has been accepted for consideration, the mechanism of which is described by the expressions (1)-(4). According to our classification, the system is a one-step directly collectible system with the collection $\Delta=\tilde{\Delta}\left(x, x^{0}, \omega\right), \quad x \in X(\omega)$.

In the study of hierarchical systems, the mathematical model (1)-(4) plays the role of an elementary system, the specification of which allows receiving theoretical and practical models of systems with many centers of decision-making. Relations in the system can be easily reconstructed by analyzing the expressions (1)-(4). The main functions of the center are to choose a preliminary solution and find the level of remuneration of the performer in the solution of the game (3)-(4).

The performer's functions are reduced to the choice of the level of solution correction in accordance with the 
designated reward and search for a corrected plan (expression (4)). The process of correcting solutions is implemented by the center and the performer jointly. This model of corrective optimization was first published in the works (Oskorbin, 1987), (Cooper and Edgett, 2006), in which its decomposition was proposed.

Let $f(x, \omega)$ be continuous by $x$ for any $\omega \in \Omega$, and the set $X(\omega)$ be compact. Then the set of values of the collection $\Delta$ coincides with the interval $\left[0-\bar{\Delta}\left(x^{0}, \omega\right)\right], \bar{\Delta}\left(x^{0}, \omega\right) \geq 0$. The center should select for each $\omega \in \Omega$ the strategy for incentives in the class of functions $\tilde{\sigma}:\left[0-\bar{\Delta}\left(x^{0}, \omega\right)\right] \rightarrow[0-1]$. Then, the decomposition of the game (3)-(4) has the form (7).

1. The management center's task is:

$$
M^{0}(\sigma, \Delta)=(1-\sigma) \Delta \rightarrow \max _{\sigma \in[0-1]}
$$

2. The performer's task is:

$$
M(\sigma, \Delta)=\sigma \Delta-\varphi(\Delta) \rightarrow \max _{\Delta \in\left[0-\bar{\Delta}\left(x^{0}, \omega\right)\right]} .
$$

3. The problems correction task is:

$$
\left|\Delta^{K}-\tilde{\Delta}\left(x^{K}, x^{0}, \omega\right)\right|=\min _{x \in X(\omega)}\left|\Delta^{K}-\tilde{\Delta}\left(x, x^{0}, \omega\right)\right|=0,
$$

Wherein $\Delta^{K}$ is an optimal strategy for the performer in the game (5)-(6).

Let us consider the applicability conditions for the corrective optimization model for research purposes, as well as for solving applied tasks. This model is adequate to real decision-making processes if the vector of the parameters $\omega \in \Omega$ unknown to the management center in the first stage is constant during the planning period. For production systems, this may be the prices for raw materials and finished products, the technological process parameters, the levels of actual labor input and materials costs in the production of intermediate and finished products, the opportunities of innovative development, and the new organizational forms of work performance.

For industrial enterprises, the following tasks can be solved using the model description:

1. Regulation of the rational level of awareness of the central management body, assessment of the loss of optimality of management in the circumstances of incomplete and asymmetric awareness of the decision-making centers.

2. Development of a system of labor compensation and incentives consistent with the objectives of the system as a whole and the market conditions of attracting labor power.

\subsection{The Functional Structure of the Personnel Management System}

The detailed model of decision-making in a hierarchical system with the involvement of the well-known provisions of both the theory of active systems (Oskorbin, 1987; Stroiteleva, 2006), and the theory of hierarchical games (Germeier, 1971; Nelson \& Winter, 1982) was considered. The general principles of personnel management in economic systems are shown.

The structure of making and implementing solutions in the considered system is shown in Figure 1. 


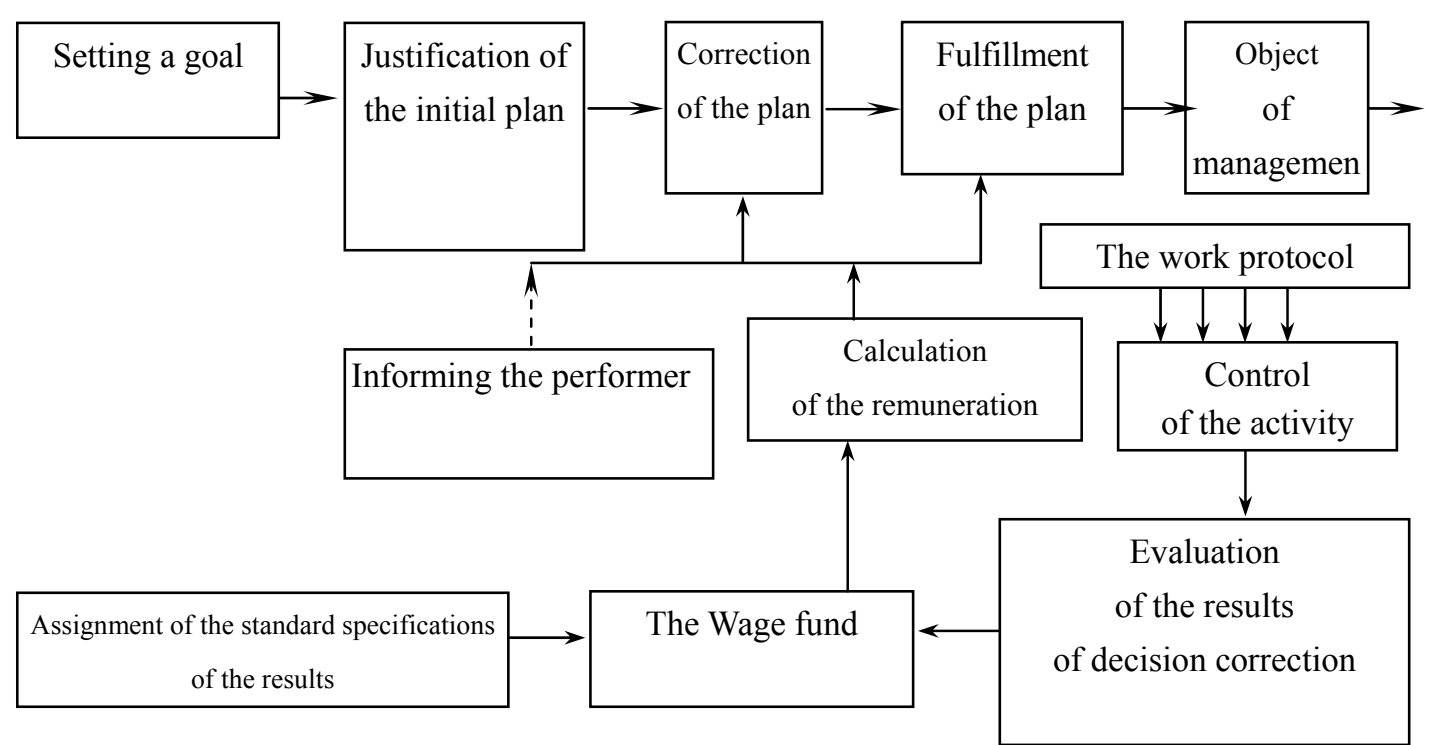

Figure 1. The structure of decision-making in a human resources management system

The traditional personnel management system (goal setting, contingency planning, information support, implementation of decisions, etc.) was supplemented by the function of the performer's activity control (Gorelik et al, 1991; Van Horne, 2009):

- control over the performance of the performer;

- assessment of the actual results;

- purposeful informing of the performer, which assumes informing the performer about the targets of management in the system and its role in this process, the management program, which is necessary for evaluation of the performance, development of standards, control procedures, methods for calculating estimates of the obtained results, the principles of reward and punishment. Informing must be performed in "human forms" and be made in advance (the figure shows information messages of this function highlighted with the dashed arrow) (Vukovic \& Miller, 2013; Higgins, 1998);

- calculation of the regulatory outcome;

- formation of the fund of labor compensation and incentive of the performer depending on the standard and actual results.

In this structure, the performer has the following functions:

- correction of the plan, which is reduced to assessment of the rational for him level of correction (the rational level of activity) in the remuneration system implemented by the center and justification of the corrective solutions;

- fulfillment (implementation) of the corrected planning solutions.

Currently, the scientific bases of management in hierarchical systems are only being formed, so this structure is not applicable to all cases of personnel management. Nevertheless, it is recommended to use this structure in practice $[4 ; 9 ; 16]$.

\section{General Principles of Modeling Undeveloped Workflows}

The problems of synthesis of personnel management for the developing simple hierarchical systems are limited to the development of specific incentive models. Three types of basic hierarchical systems are considered, in which the development is implemented by improving the equipment, changing the work technology and the staff skills. In this case, general degradation of the systems is possible (Ivanilov \& Lotov, 1979; Bogoviz et al., 2013) $[2 ; 12 ; 13]$.

As an example, we can provide the model of stimulation of the equipment assimilation processes.

Let us suppose that for $N$ periods of operation of a hierarchical system the dynamics of marginal productivities $\bar{\Delta}$ of the equipment is set by the equation 


$$
\bar{\Delta}=R_{j}\left(\bar{\Delta}_{j-1}, \tau_{j}\right), j=1, \ldots, N,
$$

wherein $\tau_{j}$ is the costs of personal time by the performer for improvement of the equipment during the period $j$.

Let us introduce the vectors $\sigma=\left(\sigma_{1}, \ldots, \sigma_{N}\right)$ of the ratios of revenue sharing (the center's strategy) and $\Delta=\left(\Delta_{1}, \ldots, \Delta_{N}\right)$ of actual equipment manufacturers.

1. The management center's task:

$$
M_{0}(\sigma, \Delta, \tau)=\sum_{j=1}^{N}\left(1-\sigma_{j}\right) T_{j} \Delta_{j} \rightarrow \max _{\substack{\sigma_{j} \in[0,1] \\ j=1, \ldots, N}}
$$

2. The performer's task:

$$
\begin{aligned}
& M(\sigma, \Delta, \tau)=\sum_{j=1}^{N}\left[\sigma_{j} T_{j} \Delta_{j}-\varphi_{j}\left(\Delta_{j}, \bar{\Delta}_{j}\right)-\alpha \tau_{j} \rightarrow \max _{0 \leq \Delta_{j} \leq \bar{\Delta}_{j}}\right. \\
& 0 \leq \tau_{j} \leq T_{j}^{0} ; \\
& j=1, \ldots, N \text {. }
\end{aligned}
$$

wherein $T_{j}$ is the duration of the period $j$;

$\alpha$ - the price of personal time of the performer;

$T_{j}^{0}-$ the fund of personal time of the performer during the period $j(j=1, \ldots, N)$. Other designations are the

same as the designations in the expressions (5)-(7).

The models (8) and (9) allow identifying the relationship in hierarchical systems, in which the equipment assimilation will provide for improvement of the system performance. We need to note that this model can be used to verify the adequacy of the incentive models, as the scientific literature contains detailed results of the study of processes of production assimilation. Besides, one may be tasked with the development of methods for identification of model parameters and numerical schemes for finding optimal strategies for the management center, which is more efficient to be carried out interactively.

\section{Applied Models of Workflows}

The basic models of workflows are based on the corrective optimization model. Research is conducted to justify the models for stimulation and study of the properties of workflows.

The form of the performer's objective function can be interpreted as an analog of "profit" of the performer who receives the difference between the labor compensation $f(v)$ and the labor input $\varphi(v)$ depending on the intensity of labor $v$ and seeks maximizing this "income". The decision-making model can be considered according to two criteria: $f(v)$ and $-\varphi(v)$ - with their simple convolution: $M(v)=f(v)-\varphi(v)$, which takes into account the criteria with equal weighting coefficients (Oskorbin, 1987; Bogoviz A. et al., 2013; Mezhov et al., 2014).

\section{Results}

In our studies, we adopt, first of all, various weighting coefficients; secondly, the behavior model is recommended to describe typical performers, as well as to analyze the stability of incentive mechanisms in deviations; thirdly, the modeling is based on the principle of "a reflecting system", which is formulated as follows: the "value" of labor compensation and the "disutility" of labor are subjective reflections of the reality for an individual and a group of individuals. The staff strives to maximize the evaluation of their payment and minimize the expected labor input. Following the principle of "a reflecting system", one needs to use in the models not an objective monetary labor compensation and amount of labor input, but the values reproduced by people, which may differ significantly from those commonly used. Within the framework of the principle of "the reflecting system", it is possible to explain many paradoxes in the economic behavior of people.

Let us consider one of the ways to assess the weighting coefficients in the objective function of a typical performer of the following type (Germeier, 1971; Oskorbin, 1987; Prahalad, 1996): 


$$
\begin{gathered}
M(v)=f(v)-\delta \cdot \varphi(v) ; v \in[0, \bar{v}] . \\
M(v)=f(v)-\delta \cdot \varphi(v) ; v \in[0, \bar{v}]
\end{gathered}
$$

Let us assume that normal intensity $v_{H}$ has been found for this workflow by the method of labor rating. Usually, when rating labor, piecework payment is used, i.e. $f(v)=\frac{P_{0} v}{v_{H}}$ where $P_{0}$ is the wage rate, the performance rate $v_{H}$. There occurs a possibility to estimate the parameter $\delta$ from the equality of the real and estimated behavior.

Let it be $\tilde{v}(\delta)=\arg \max \{f(v)-\delta \varphi(v) / v \in[0, \bar{v}]$.

Then the required quantity $\delta$ is the root of the equation $\tilde{v}(\delta)=v_{H}$.

If labor input is proportional to the negative logarithm $-\ln \left(1-\frac{v}{\bar{v}}\right)$, then $\delta_{r}=P_{0}\left(1 / K_{H}-1\right)$ wherein $K_{H}=v_{H} / \bar{v}$; if the functions $v /(\bar{v}-v)$, then $\delta_{2}=P_{0}\left(1-K_{H}\right)^{2} / K_{H}$. In this case, there is no need to identify the labor input and use the principle of a reflecting system, because the field proven models of labor rating are applied for the standard process. In the future, the following two functions of labor input are often used in specific models (Bogoviz et al, 2013), (Oskorbin, 1989):

$$
\varphi_{n}(v)=-\delta_{n} \ln \left(1-\frac{v}{\bar{v}}\right) \text { and } \varphi_{2}(v)=\delta_{2} v /(\bar{v}-v)
$$

For the payment and incentives models, we should suggest indicators, the values of which would make it possible to estimate the performance capability of models and their conformance to the socioeconomic recommendations. The indicators of compliance of payment to the labor expended and the normality of the labor input in the workflow act as informative ones. In practice, one needs to have the margin values of indicators that could be involved in the evaluation of performance of a certain labor compensation plan. The practical application of the research results of theoretical models of workflows solves the problems of parameters identification, assessment of the adequacy and efficiency of simple compensation and incentive models, in which it is possible to use the results of the labor rating. All of the above aspects for complex models of developing hierarchical systems require additional research (Chesbrough et al., 2006) $[5 ; 19 ; 20]$.

Let us consider the specific models of simple hierarchical systems, as listed in Table 1. We will provide the research results and methods for specific cases.

Table 1. Digest of the results of workflows modeling

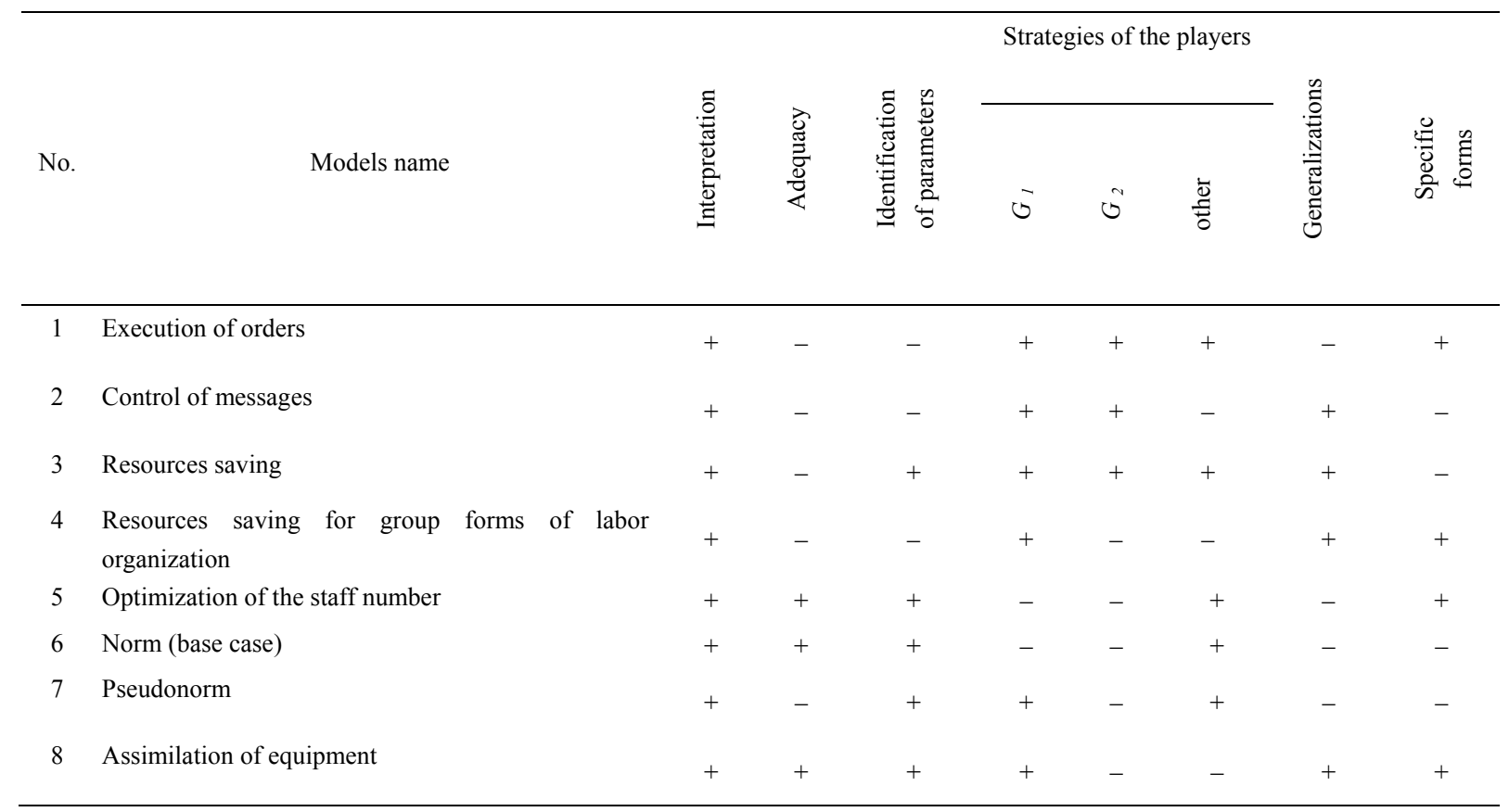


9 Revision of pseudonorms at the initiative of the management center

10 Revision of pseudonorms at the initiative of the performer

11 Revision of the norms depending on the actual performance

12 Management of skills

The model of messages control is a particular case of the model (10). Let the performer who is tasked to report the $s_{0}$ state of the system distorts the information to the value $s$. Let us input $\varepsilon=\left\|s^{0}-s\right\|$ - the message error and consider the function $M(P, \varepsilon)$. Let the management center imposes a fine on the performer proportional to the value of errors: $(f(\varepsilon)=d \varepsilon)$ and the labor input function is as follows: $\varphi(\varepsilon)=b / \varepsilon$, then $(22) V=[0, \bar{\varepsilon}]$, wherein $\bar{\varepsilon}$ is the margin message error, for which the administrative methods of management are applied, and $M(P, \varepsilon)=P \varepsilon+\frac{B}{\varepsilon}$, wherein $B=\frac{b}{d}$ is the only parameter of the performer's model. Let us consider the optimal strategies of the players and continue to interpret the model. Analysis of the model shows that the game $G_{l}$, which is common in the hierarchical games theory, in this case provides a system of relations between the players, which is not practicable because the error $\varepsilon$ is not rated by the center.

\section{Discussion}

Let us consider the strategies of the players in the game (10), resulting from the mechanism $G_{2}$.

Assertion 1. Let us suppose that in the model of messages control $M_{0}(P, \varepsilon)$ increases monotonically by $P$ and $\varepsilon$, and the performer is friendly to the goals of the center; $\bar{\varepsilon}>0, B>0$. The strategy of the center $\tilde{P}(\varepsilon)$ establishes dependence of the control frequency $P$ on the error of the message that appears in the system because the targets of the center and the performer do not coincide. There is also visible impracticability of the strategy, as in any real situation, it is impossible to accurately verify the equality $\varepsilon=\varepsilon^{0}$. However, in most real systems, it is possible to replace this equality with the strict inequality $\varepsilon \leq \varepsilon^{0}$, and this change shows that the function of the performer's punishment for being too diligent is not implemented by the organizational structure. This observation may not apply to simulators of the operational capabilities of the staff. In this paper (Bogoviz et al, 2013), the peculiar features of the organizational mechanism operation are considered, and it is shown that the center requires two systems of message control and the accuracy requirements for these systems are formulated. The conclusions are valid for all models of messages control, in which the function $f(\varepsilon)$ increases monotonically by $\varepsilon$, and $\varphi(\varepsilon)$ meets the following conditions: $\varphi^{\prime}(\varepsilon)<0 ; \varphi^{\prime \prime}(\varepsilon)<0 ; \quad \lim \varphi(\varepsilon)=\infty$. The models for fulfillment of instructions are found in particular cases of expression of the optifna ${ }^{0} G_{1}$ and the $G_{2}$ strategies of the center. It is shown that the situation $\left(P^{*}=0, v^{*}=0\right)$ is the minimax solution and an equilibrium for the game (17), and also the behavior of the players according to these strategies does not correspond to real systems.

Models of resource saving are a particular case of incentive models at corrective optimization. The essence of the models is that from the value of the saved resource $\left(L\left(v_{0}-v\right)\right.$, where $P$ is the price for a resource; $v_{0}$ is the usage of a resource during the base period; $v$ is the consumption of the resource in the saving mode) a portion equal to $\lambda\left(v_{0}-v\right)$ is allocated to reward the performers. Here is an example of a model with a team form of the bonuses distribution, when the center controls the total consumption of the resource: $v=v_{1}+v_{2}+\ldots+v_{n}$, wherein $v_{i}$ is the consumption of the resources of the performer $i$. A simple hierarchical system of relations emerges, the analysis of which reveals the pattern of team forms of labor compensation and incentives.

1. The center's task:

$$
M_{0}(\lambda, v)=(L-\lambda)\left(v_{0}-v\right) \rightarrow \max _{0 \leq \lambda \leq L} .
$$

2. The task of all performers: 


$$
M\left(\lambda_{1} v_{1}, \ldots, v_{n}\right)=\sum_{i=1}^{n}\left[\lambda\left(v_{0}-v\right)-\varphi_{i}\left(v_{i}\right)\right] \rightarrow \max _{\substack{v_{i} \geq v_{0 i} \\ i=1, \ldots, n .}}
$$

Research shows that for quadratic, logarithmic functions of labor input the aggregative form of the game (11)-(12) is easily introduced, where the center considers the team of performers as a single player. Aggregation is possible under the condition of the team integrity, when each person maximizes the group criterion. Let us note that the coalition criterion postulated in the game theory requires support mechanisms in the reality.

It is easy to show that in the game (11)-(12) it is advantageous for one of the players not to participate in the resource-saving mode and keep gaining part of the group bonus at the same time. Focusing on the self-organization of the team members, the work formulates the conditions of integrity of the team. The conditions consist in the fact that, before subjective evaluations, all the performers, first of all, recognize the method of allocating bonuses as fair, and, secondly, they know that every performer really maximizes his objective function in the game (11)-(12).

Let $P=\lambda\left(v_{0}-v\right)$ be the aggregate bonus of the team, and $P_{i}, i=1, \ldots, n$ be its distribution $\left(P=P_{1}+\ldots+P_{n}\right)$. Any performer subjectively evaluates the amount of the required bonus of the performer $j$ within the accuracy to the set $\left[P_{i}^{j}, \bar{P}_{i}^{j}\right]$. Then the first condition is satisfied if $P_{i}^{j} \leq P_{i} \leq \bar{P}_{i}^{j}, i, j=1, \ldots, n$. In this case, consistency of the views of the team members is required, i.e. the validity of the conditions:

$$
P_{i}=\max _{1 \leq j \leq n} P_{i}^{j} \leq \bar{P}_{i}=\min _{1 \leq j \leq n} \bar{P}_{i}^{j}, i=1, \ldots, n .
$$

Analysis of these conditions should be carried out under the assumption of the integrity of the team.

Let us consider the second condition. Let $P_{F_{I}}$ be the actual contribution of the performer $i$ in the total amount of the bonus, and $\left[\underline{P}_{F_{i}}^{j}, \bar{P}_{F_{i}}^{j}\right]$ be its evaluation by the performer $j$. The team will be integrated if for any pair $(i, j)$ the inequalities are valid: $\underline{P}_{F_{i}}^{j} \leq P_{F_{i}} \leq \bar{P}_{F_{i}}^{j}$. The defined criteria of integrity and the analysis results allow explaining some of the paradoxes of the teams' performance and may be useful in practice.

In the proposed conditions of integrity and with the given mechanisms for their verification, the number of control pairs in the team is equal to the square of the performers number, therefore, the costs of self-control also increase. The problem of optimizing the size of the team taking into account the conditions for minimum costs of control emerges. The task in the simplest case is stated as follows (Oskorbin, 1987).

Let us consider the group of situations in a hierarchical system, in which the control mechanisms use norms and standard specifications. The simplest model is the model of piecework payment, which has the following form (the pseudonorms model).

1. The center's task:

$$
M_{0}^{H}(H, \Delta)=\left(1-\frac{P_{0}}{H}\right) \Delta \rightarrow \max _{H \geq P_{0}}
$$

2. The task of a typical performer:

$$
M^{H}(H, \Delta)=\frac{P_{0}}{H} \Delta-\varphi(\Delta) \rightarrow \max _{0 \leq \Delta \leq \bar{\Delta}} .
$$

In this model, $H$ is the pseudonorm (the center's strategy);

$\bar{\Delta}$ is the potential performance rate;

$P_{0}$ is the wage rate.

In the works (Germeier, 1971) and (Stroiteleva, 2006), the method for personnel management obtained from (13)-(14) is called the mechanism of "soft" and "hard" rules. Analysis of the model and plans of labor compensation shows that in this case the replacement of the pseudonorm $H$ with $\Delta_{H}$ - the performance rate calculated according to the methods of work - is a good approximation of the optimal strategy of the center in the game (13)-(14), as illustrated by the following assertion. 
Assertion 2. Let us suppose that in (13)-(14), the function $\varphi(\Delta)$ is logarithmic, $P_{0} K_{H}<\bar{\Delta}$, and $b=P_{0}\left(1 / K_{H}-1\right)$, wherein $K_{H}=\Delta_{H} / \bar{\Delta}$.

Then $M_{0}\left(H^{*}, \Delta^{*}\right)-M_{0}\left(\Delta_{H}, \tilde{\Delta}\left(\Delta_{H}\right)\right)=\bar{\Delta}\left(\sqrt{1-K_{H}}-\sqrt{\frac{P_{0}}{\bar{\Delta} K_{H}}}\right)^{2}$ is the assessment of optimality losses of the mechanism of the norm with respect to the mechanism that implements the optimal pseudonorm in the strategies $G_{l}$. The dependence of the optimality losses is presented in Table 2. This result shows a thoroughly practiced implementation of the piece rate wage plan.

Table 2. The relative optimality losses caused by use of performance rate as the strategy of the center (the model of standards)

\begin{tabular}{llllllllll}
\hline \multirow{2}{*}{$K_{H}$} & \multicolumn{7}{c}{$P_{0} / \Delta_{H} P_{0} / \Delta_{H}$} \\
\cline { 2 - 10 } 0.1 & 0.100 & 0.200 & 0.300 & 0.400 & 0.500 & 0.600 & 0.700 & 0.800 & 0.900 \\
0.2 & 0.334 & 0.200 & 0.120 & 0.069 & 0.035 & 0.014 & 0.003 & 0.000 & 0.003 \\
0.3 & 0.271 & 0.152 & 0.083 & 0.042 & 0.017 & 0.004 & 0.000 & 0.003 & 0.013 \\
0.4 & 0.210 & 0.107 & 0.051 & 0.020 & 0.005 & 0.000 & 0.004 & 0.014 & 0.030 \\
0.5 & 0.153 & 0.068 & 0.025 & 0.006 & 0.000 & 0.005 & 0.017 & 0.035 & 0.058 \\
0.6 & 0.100 & 0.034 & 0.007 & 0.000 & 0.006 & 0.020 & 0.042 & 0.069 & 0.100 \\
0.7 & 0.054 & 0.010 & 0.000 & 0.007 & 0.025 & 0.051 & 0.083 & 0.120 & 0.161 \\
0.8 & 0.017 & 0.000 & 0.010 & 0.034 & 0.068 & 0.107 & 0.152 & 0.200 & 0.251 \\
0.9 & 0.000 & 0.017 & 0.054 & 0.100 & 0.153 & 0.210 & 0.271 & 0.334 & 0.400 \\
\hline
\end{tabular}

Table 3 shows corrections for the case if the management decides to use optimal pseudonorms in the piece-rate wage plan. This result is considered important from the point of view of the adequacy of the stimulation model (5), (6). We need to note that the models (13)-(14) coincide with it if we adopt $\sigma=\frac{P_{0}}{H}$.

Table 3. Coefficients of $d$ correction of the performance rates at calculating the optimal pseudonorm

\begin{tabular}{ccccccccccc}
\hline & \multicolumn{7}{c}{$P_{0} / \Delta_{H} P_{0} / \Delta_{H}$} \\
\cline { 2 - 10 } & 0.100 & 0.200 & 0.300 & 0.400 & 0.500 & 0.600 & 0.700 & 0.800 & 0.900 \\
0.1 & 0.333 & 0.471 & 0.577 & 0.667 & 0.745 & 0.816 & 0.832 & 0.943 & 1.000 \\
0.2 & 0.354 & 0.500 & 0.612 & 0.707 & 0.791 & 0.866 & 0.935 & 10,000 & 1.061 \\
0.3 & 0.378 & 0.35 & 0.655 & 0.756 & 0.845 & 0.926 & 1.000 & 1.069 & 1.134 \\
0.4 & 0.408 & 0.577 & 0.707 & 0.816 & 0.913 & 1.000 & 1.080 & 1.155 & 1.225 \\
0.5 & 0.447 & 0.632 & 0.775 & 0.894 & 1.000 & 1.095 & 1.183 & 1.265 & 1.342 \\
0.6 & 0.500 & 0.707 & 0.866 & 1.000 & 1.118 & 1.225 & 1.323 & 1.414 & 1.500 \\
0.7 & 0.577 & 0.816 & 1.000 & 1.555 & 1.291 & 1.414 & 1.528 & 1.633 & 1.732 \\
0.8 & 0.707 & 1.000 & 1.225 & 1.414 & 1.581 & 1.732 & 1.671 & 2.000 & 2.121 \\
0.9 & 1.000 & 1.414 & 1.732 & 2.000 & 2.236 & 2.449 & 2.646 & 2.828 & 3.000 \\
\hline
\end{tabular}

The model of equipment assimilation (8), (9) can be studied by numerical methods in strategies $G_{l}$ in the variant of the pseudonorms mechanism. The performer's task at fixed pseudonorms of assimilation is solved using dynamic programming $[14 ; 16 ; 17]$.

Let us consider special models of equipment assimilation (see Table 1). The model of pseudonorms revision at the initiative of the center is of the form: 


$$
\begin{aligned}
& M_{0}(H, \Delta, \tau)=\left(\frac{P_{0}}{H_{0}}-\frac{P_{0}}{H}\right) \Delta T \rightarrow \max _{H \geq H_{0}} ; \\
& M(H, \Delta, \tau)=\frac{P_{0} T \Delta}{H}+\delta T \ln \left(1-\frac{\Delta}{O}\right)-\alpha \tau \rightarrow \max _{\Delta_{0} \leq \Delta \leq O} ; \\
& 0 \leq \tau \leq T_{0} \\
& O=O_{0}+b \tau ; \frac{P_{0} T \gamma}{H}-P_{0 T} \geq \alpha \tau,
\end{aligned}
$$

where $H_{0}=\Delta_{0}=O_{0} K_{H}$ is the pseudonorm and the actual effect of solutions correction for the base period;

$O_{0}, O$ - the potentially achievable outcomes of the solutions correction;

$$
\gamma=\frac{b T}{T_{0}} \quad \text { - the model parameter. }
$$

The remaining values are described in the expressions (13), (14) (we assume that $N=1$, the index $j$ is omitted). The model uses the linear function of the system development and accepts the conditions of full recovery of the performer's expenses for development of the system (expression (17)). Let us note that (15)-(17) is a game with forbidden situations. Let us solve it.

Assertion 3. Let the performer be friendly to the goals of the center, and in the description of the game situation (15)-(17), the parameters at the strictly positive $\alpha, P_{0}, \delta, T_{0}$ meet the condition: $P_{0} \gamma \geq \alpha$. Then the set of strategies of the center is not empty, and the optimal strategy has the form:

$$
H^{*}=\min \left(\frac{P_{0} b T_{0}}{\alpha \frac{T_{0}}{T}+\delta \ln \left(1-\gamma \frac{T_{0}}{T}\right)}, \frac{O_{0}\left(1+\gamma \frac{T_{0}}{T}\right) P_{0}}{P_{0}+\delta+\alpha \frac{T_{0}}{T}}\right)
$$

The analysis of the model and the solution shows that if $P_{0} \gamma<\alpha$, then stimulation of the equipment assimilation is impractical. Analysis and interpretation of the optimal pseudonorm show that it stimulates overstressed labor, which corresponds to the experience.

Let us use similar schemes to consider some mechanisms for revision of pseudonorms depending on the achieved effect of correction at the initiative of the performer.

The pseudonorm varies according to the formula:

$$
H=(1-\beta) H_{0}+\beta \Delta, \quad \beta \in[0,1]
$$

The article considers the problems of mathematical modeling of workflows and relations in the "employee employer" system. In this approach, the employer is characterized by the attributes that are not taken into account in other approaches. Firstly, it cannot (or will not) describe the production and management processes of the lower levels; secondly, the employer must provide controllability of the system (the optimal operation of the production system as a whole).

\section{Conclusion}

The main scientific results of the research are as follows:

1. A new method for modeling active elements in production systems has been suggested, which includes formulation of managerial tasks in hierarchical active systems in the circumstances of unidentifiable reactions of the lower levels (employees), development of generalized and private models of personnel management in the systems of production type, and numerical solution of the respective mathematical problems.

2. A set of specific mathematical models of stimulation of workflows performers has been developed.

The provided results can be used for the real designing and model studies of management mechanisms in production systems. 


\section{References}

Bogoviz, A. V. (2013). Developing a Systemic Labor Motivation in Enterprises. World applied sciences journal, 25(10), 1429-1431.

Bogoviz, A. V., \& Stroiteleva, E. V. (2012). Management of the economic condition of an enterprise using an information system. Bulletin of the Altai Academy of Economics and Law, 2, 52-55.

Bogoviz, A. V., Vukovich G. G., \& Stroiteleva, T. G. (2013). Modeling the Workflow One of the Tasks of Strengthening of Positive Trends in the Economic Growth of the Industrial Enterprises of the Region. World Applied Sciences Journal, 8(25), 1222-1225.

Bogoviz, A. V., Vukovich, G. G., \& Stroiteleva, T. G. (2013). Motivation of Staff in the Corporate Sector Industry. World Applied Sciences Journal, 25(10), 1423-1428.

Chesbrough, H. W. (2006). Open Innovation: Researching a New Paradigm. Oxford: Oxford University Press,.

Cooper, R. G. (2006). Edgett, S.J. Stage-Gate and the critical success factors for new product development. BP Trends, 7.

Dosi, G., \& Teece, D. (1998). Organizational competencies and the boundaries of the firm. In Arena, R., \& Longhi C. (Eds.), Markets and Organization (pp. 281-301). New York: Springer-Verlag. http://dx.doi.org/10.1007/978-3-642-72043-7_12

Freeman, Cr. (1988). Unemployment and Technical Innovation: A Study of Long Waves in Economic Development. Cambridge, MA: Harvard University Press.

Germeier, J. B. (1971). Introduction to Operations Research (pp. 164). Moscow: Nauka.

Gorelik, V. A. (1991). An analysis of conflict situations in the control systems (pp. 208). Moscow: Radio and Communications,.

Higgins, R. (1998). Analysis for Financial Management (5th ed.). Irwin.

Ivanilov, Y. P., \& Lotov, A. V. (1979). Mathematical models in economics (pp. 304). Moscow: Nauka.

Mezhov, S. (2014). Theoretical and Methodological Bases of Forming the Operational and Innovation Program of the Company. Advances in Natural and Applied Sciences, 8(5), 451-457.

Nelson R., \& Winter, S. (1982). An Evolutionary Theory of Economic Change. Cambridge, MA: Harvard University Press.

Oskorbin, N. M. (1987). Operations Research and Game Theory in the elementary exposition: The text of the lectures (pp. 62). Barnaul Univ Alt. Press.

Oskorbin, N. M. (1989). Some Aspects of Optimization of Decision Support System. Volume of Abstracts International School: Seminar Optimization Methods and Their Applications (pp. 66-67). Baikal.

Prahalad, C. K. (1996). Competing for the future. Harvard Business School Press.

Stroiteleva, T. G. (2006). Problems of personnel policies machine-building enterprises of the Altai Territory: monograph (pp. 188). Barnaul Univ Alt. Press.

Van, H. J. (2009). Fundamentals of Financial Management (13th ed.). Prentice Hall.

Vukovich, G. G., \& Miller, A. I. (2013). Economic-mathematical methods for assessing the functioning of the corporate structures of the region. Journal of Economic Integration, 3(60), 50-54.

\section{Copyrights}

Copyright for this article is retained by the author(s), with first publication rights granted to the journal.

This is an open-access article distributed under the terms and conditions of the Creative Commons Attribution license (http://creativecommons.org/licenses/by/3.0/). 